\title{
MHD FLOW OF LONGITUDINAL AND TORSIONAL OSCILLATIONS OF A CIRCULAR CYLINDER WITH SUCTION IN A COUPLE STRESS FLUID
}

\author{
G. NAGARAJU* \\ Department of Mathematics, GITAM University, Hyderabad Campus \\ Rudraram, Medak District, Andhra Pradesh, INDIA \\ E-mail: naganitw@gmail.com \\ J.V. RAMANA MURTHY \\ Department of Mathematics \\ National Institute of Technology \\ Warangal-506004, (A.P), INDIA \\ E-mail: jvrjosyula@yahoo.co.in
}

\begin{abstract}
In this paper, we consider the flow an incompressible electrically conducting couple stress fluid generated by performing longitudinal and torsional oscillations of a porous circular cylinder subjected to constant suction/injection at the surface of the cylinder and in the presence of a radial magnetic field. A finite difference method is proposed to analyze the velocity components, in an infinite expansion of a couple stress fluid under vanishing couple stresses on the boundary. The effects of the magnetic parameter, couple stress parameter, Reynolds number, the ratio of couple stress viscosities parameter and suction parameter on velocity components and drag are discussed and shown graphically.
\end{abstract}

Key words: longitudinal and torsional oscillations, drag, suction, radial magnetic field.

\section{Introduction}

The problem of longitudinal and torsional oscillations of a cylinder in fluids has considerable practical applications involving mixing, oil drilling and towing operations. Important applications of these kinds of flows are in oil industries where the knowledge of the behavior of oil flowing through a pipeline and oscillating in a cylindrical rod is required and in oceanic engineering where information on the drag forces on rods is useful.

Ramkissoon et al. (1990) studied the internal flow due to longitudinal and torsional oscillations of a viscous fluid and they derived an analytical expression for velocities, shear stresses and drag on the cylinder. Majumdar et al. (1991) obtained an exact solution for an infinite rod undergoing both longitudinal and torsional oscillations in a polar fluid. They presented the effect of micropolar parameters on the microrotation and velocity fields graphically. Calmelet-Eluhu et al. (1998) studied the internal flow of a micropolar fluid inside a circular cylinder subject to longitudinal and torsional oscillations and they showned the effect of micropolar fluid on two components velocity field through graphs. Owen and Rahman (2006) studied the same type of flow with an Oldroyd-B liquid.

The flow through porous boundaries is of great importance both in technological as well as biophysical flows. Examples are found in soil mechanics, transpiration cooling, food preservation, cosmetic industry, blood flows and artificial dialysis. Bansal (1967) studied the steady flow of a viscous fluid through a porous circular pipe with small suction at the porous wall and the effects of various viscous parameters and suction parameter on the axial pressure gradient, axial and radial velocity components and skin friction.

\footnotetext{
* To whom correspondence should be addressed
} 
Agarwal (1969) studied the steady flow of a viscous incompressible fluid through a circular pipe with normal suction at the porous wall and he showned the effects of various viscous parameters on the axial pressure gradient, axial velocity and shear stresses graphically. Tsangaris et al. (2007) obtained the exact solution for the unsteady laminar axi-symmetric flow of a viscous liquid in a straight pipe of circular cross section with varying injection and/or suction applied at a porous wall. This is a generalization of Terril's problem (1965). Muthu et al. (2008) studied the peristaltic motion of a micropolar fluid with viscoelastic or elastic wall properties and observed the influence of wall properties for various values of micropolar fluid parameters. The problem is studied numerically by finite difference methods. In recent years, several simple flow problems associated with classical hydrodynamics have received attention within the more general context of magneto hydrodynamics (MHD). Several investigators have extended many of the available hydrodynamic solutions to include the effects of the magnetic field for those cases when the fluid is electrically conducting. Singh and Syed Ali Tahir Rizvi (1964) investigated the impulsive motion of a viscous liquid contained between two concentric circular cylinders in the presence of a radial magnetic field. Hughes and Young (1966) obtained the velocity distribution and magnetic field for a viscous incompressible conducting fluid between two coaxial rotating cylinders under the influence of a uniform radial magnetic field of the form $B / r$ throughout the fluid region. Ramana Murthy and Bahali (2010) studied the unsteady MHD flow of a micropolar fluid through a porous circular pipe with constant suction/injection. They observed that as the magnetic parameter increases the skin friction increases and numerically decreases with increasing values of couple stress parameter. Srinivasacharya et al. (2012) studied an analytical solution of a mixed convection flow of a couple stress fluid between two concentric cylinders with Hall and Ion effects.

The couple stress fluid theory developed by Stokes (1966) represents the simplest generalization of the classical viscous fluid theory that sustains couple stresses and the body couples. The important feature of these fluids is that the stress tensor is not symmetric and their accurate flow behavior cannot be predicted by the classical Newtonian theory. The fluids consisting of rigid, randomly oriented particles suspended in a viscous medium, such as blood, lubricants containing small amount of polymer additives, electro-rheological fluids and synthetic fluids are examples of these fluids. Recently longitudinal and torsional oscillations of a circular cylinder with suction in a couple stress fluid was studied by Ramana Murthy et al. (2010).

The objective of this paper to investigate the magnetic field and suction effect on the flow of a couple stress fluid generated by a circular cylinder performing longitudinal and torsional oscillations and subjected to suction velocity at the surface.

\section{Formulation of the problem}

Consider a porous circular cylinder of radius ' $a$ ' in an infinite expansion of a couple stress fluid. The cylinder is subjected to torsional oscillations, $\operatorname{Exp}\left(i \omega_{1} t\right)$ and longitudinal oscillations, $\operatorname{Exp}\left(i \omega_{2} t\right)$ with amplitudes $q_{0} \sin \beta_{0}, q_{0} \cos \beta_{0}$ along the respective directions where $\omega_{1}$ is the frequency of the torsional oscillations, $\omega_{2}$ is the frequency of the longitudinal oscillations, $q_{0}$ is the magnitude of the oscillations and $\beta_{0}$ is the angle between the direction of torsional oscillations and the base vector $\boldsymbol{e}_{\theta}$. i.e. The cylinder oscillates with velocity as given by the expression $\boldsymbol{Q}_{\Gamma}=\mathrm{q}_{0}\left(\sin \beta_{0} \boldsymbol{e}^{i \omega_{1} \tau} \boldsymbol{e}_{\theta}+\cos \beta_{0} \boldsymbol{e}^{i \omega_{2} \tau} \boldsymbol{e}_{z}\right) \cdot u_{0}$ is a suction or injection velocity on the surface of the porous cylinder. A cylindrical polar coordinate system is considered with the $z$ axis along the axis of the cylinder and origin on the axis. Let $R, \theta$ and $Z$ denote the radial, azimuthal and axial coordinates, respectively, of a point in the region of flow. Now we consider the flow generated in the couple stress fluid due to the oscillations of the cylinder and the flow is subject to a magnetic field along the radial direction and no external electric field is applied. We assume that the induced magnetic field is much smaller than the externally applied magnetic field. Assume that the magnetic Reynolds number is very small, so that the induced magnetic field and electric field produced by the motion of the electrically conducting fluid are negligible. The physical model illustrating the problem under consideration is shown in Fig.1. 


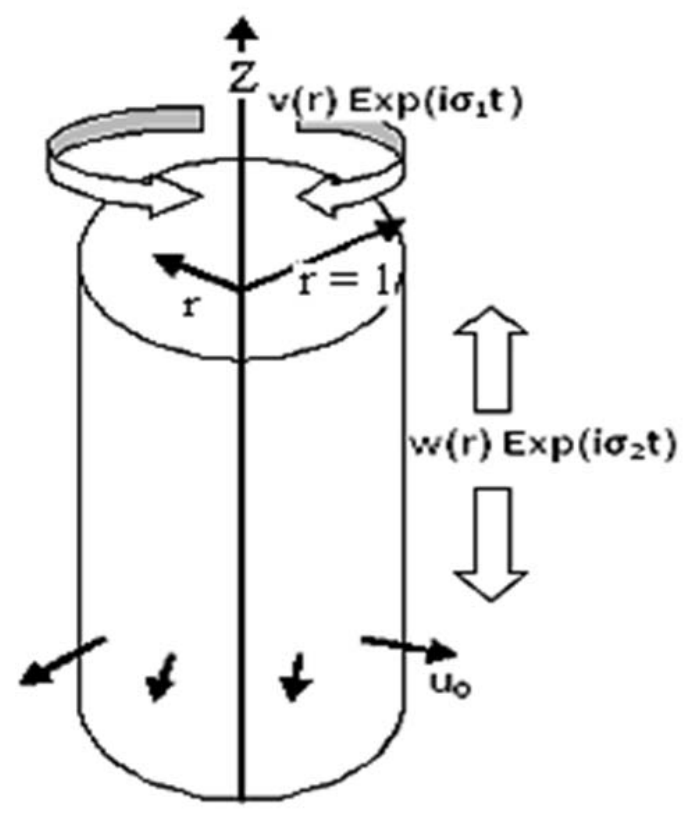

Fig.1. Geometrical representation of the problem - non-dimensional form.

After neglecting body forces and body couples, the condition of incompressibility and the equation motion for the couple stress fluid, as given by Stokes (1966) are

$$
\begin{aligned}
& \nabla_{1} \cdot Q=0, \\
& \rho\left(\frac{\partial Q}{\partial \tau}+Q \cdot \nabla_{1} Q\right)=-\nabla_{1} P-\mu \nabla_{1} \times \nabla_{1} \times Q-\eta \nabla_{1} \times \nabla_{1} \times \nabla_{1} \times \nabla_{1} \times Q+J \times B
\end{aligned}
$$

where $Q$ is the velocity vector, $P$ is the fluid pressure, $\rho$ is density, $\tau$ is time, $\mu$ is viscosity and $\eta$ is the couple stress viscosity coefficient. The current density $J$, magnetic field $B$ and electric field $E$ are related by Maxwell's equations

$$
\nabla_{l} \times E=\frac{\partial B}{\partial t}, \quad \nabla_{l} \cdot B=0, \quad \nabla_{l} \times B=\mu^{\prime} J, \quad \nabla_{l} \cdot J=0 \quad \text { and } \quad J=\sigma_{e}(E+Q \times B)
$$

where $\nabla_{l}$ is the dimensional gradient, $\sigma_{e}$ is electrical conductivity and $\mu^{\prime}$ is the magnetic permeability.

Let us introduce the following non dimensional scheme

$$
\begin{aligned}
& \sigma_{1}=\frac{\omega_{1} a}{q_{0}}, \quad \sigma_{2}=\frac{\omega_{2} a}{q_{0}}, \quad r=\frac{R}{a}, \quad q=\frac{Q}{q_{0}}, \quad p=\frac{P}{\rho q_{0}^{2}}, \\
& t=\frac{q_{0}}{a} \tau, \quad n_{1}=\frac{u_{0}}{q_{0}}, \quad u=\frac{U}{q_{0}}, \quad v=\frac{V}{q_{0}}, \quad w=\frac{W}{q_{0}} .
\end{aligned}
$$

By the non-dimensional scheme in Eqs (2.1) and (2.2), the equations for the flow are transformed to the following non-dimensional form. 


$$
\begin{aligned}
& \nabla \cdot q=0 \\
& \operatorname{Re}\left(\frac{\partial q}{\partial t}+q \cdot \nabla q\right)=-\operatorname{Re} \nabla p-\nabla \times \nabla \times q-S \nabla \times \nabla \times \nabla \times \nabla \times q-\frac{H a^{2}}{r^{2}}\left(q-u e_{r}\right)
\end{aligned}
$$

where $\operatorname{Re}=\frac{\rho q_{0} a}{\mu}=$ Reynolds number, $S=\frac{\eta}{\mu a^{2}}=$ couple stress parameter and $H a=\sqrt{\frac{\sigma_{e} B_{0}^{2}}{\mu}}=$ magnetic parameter.

In view of the oscillating boundary, the flow is assumed to be axially symmetric for slow velocities. Since the cylinder is of infinite length, the flow variables are independent of the axial variable $Z$. Hence the velocity is a function of the radial distance $r$ and time $t$ and it is assumed in the form

$$
q=u(r) e_{r}+v(r) e^{i \sigma_{1} t} e_{\theta}+w(r) e^{i \sigma_{2} t} e_{z}
$$

where $u=$ suction velocity in the radial direction is taken as $n_{l} / r$ to satisfy the incompressibility condition. (Pontrelli, 1997). Here $n_{l}$ is the suction parameter defined as $n_{l}=\frac{u_{0}}{q_{0}}$.

Equation (2.4) will yield the following three scalar equations in the directions of base vectors

$$
\begin{aligned}
& \frac{d p}{d r}=\frac{n_{1}^{2}}{r^{3}}+\frac{v^{2}}{r} e^{2 i \sigma_{1} t}, \\
& \operatorname{Re}\left(i \sigma_{1} v+\frac{n_{1}}{r}\left(v^{\prime}+\frac{v}{r}\right)\right)=-S D^{4} v+D^{2} v-\frac{H a^{2}}{r^{2}} v, \\
& \operatorname{Re}\left(i \sigma_{2} w+\frac{n_{1}}{r} w^{\prime}\right)=\left(\frac{1}{r} w^{\prime}+w^{\prime \prime}\right)-S\left(w^{j v}+\frac{2}{r} w^{\prime \prime}-\frac{1}{r^{2}} w^{\prime \prime}+\frac{1}{r^{3}} w^{\prime}\right)-\frac{H a^{2}}{r^{2}} w
\end{aligned}
$$

where

$$
D^{2} v=v^{\prime \prime}+\frac{1}{r} v^{\prime}-\frac{1}{r^{2}} v \quad \text { and } \quad D^{4} v=v^{i v}+\frac{2}{r} v^{\prime \prime \prime}-\frac{3}{r^{2}} v^{\prime \prime}+\frac{3}{r^{3}} v^{\prime}-\frac{3}{r^{4}} v
$$

Using $D^{2} v$ and $D^{4} v$ in Eq.(2.7), we get ${ }^{\prime}$

$$
v^{i v}+\frac{2}{r} v^{\prime \prime \prime}+\left(a_{1}-\frac{3}{r^{2}}\right) v^{\prime \prime}+\left(\frac{a_{2}}{r}+\frac{3}{r^{3}}\right) v^{\prime}+\left(a_{3}+\frac{a_{4}}{r^{2}}-\frac{3}{r^{4}}\right) v=0
$$

where

$$
a_{1}=-\frac{1}{S}, \quad a_{2}=\frac{\operatorname{Re} n_{1}-1}{S}, \quad a_{3}=\frac{\operatorname{Re} i \sigma_{1}}{S} \quad \text { and } \quad a_{4}=\frac{\operatorname{Re} n_{1}+H a^{2}+1}{S} .
$$

Similarly we write Eq.(2.8) as

$$
w^{i v}+\frac{2}{r} w^{\prime \prime \prime}+\left(b_{1}-\frac{1}{r^{2}}\right) w^{\prime \prime}+\left(\frac{b_{2}}{r}+\frac{1}{r^{3}}\right) w^{\prime}+\left(b_{3}+\frac{b_{4}}{r^{2}}\right) w=0
$$

where

$$
b_{1}=-\frac{1}{S}, \quad b_{2}=\frac{\operatorname{Re} n_{1}-1}{S} \quad b_{3}=\frac{\operatorname{Re} i \sigma_{2}}{S} \quad \text { and } \quad b_{4}=\frac{H a^{2}}{S} .
$$


Now Eqs (2.9) and (2.10) are solved for $v$ and $w$ under the conditions given as follows.

$$
\begin{aligned}
& v(l)=\cos \beta_{0} \quad \text { and } \quad w(1)=\sin \beta_{0} \text { (no slip condition), } \\
& D^{2} v=0 \quad \text { and } \quad w^{\prime \prime}-e \frac{1}{r} w^{\prime}=0 \quad \text { where } \quad e=\frac{\eta^{\prime}}{\eta} \quad \text { on } \quad r=1
\end{aligned}
$$

(vanishing of couple stresses on the boundary).

As $r \rightarrow \infty$, the fluid is at rest and boundary conditions can be taken as

$$
v=w=D^{2} v=w^{\prime \prime}-e \frac{1}{r} w^{\prime}=0=\frac{d}{d r}(r v)=0 \quad \text { on } \quad r=\infty .
$$

\section{Finite difference method}

In view of the complicated nature of two Eqs (2.9) and (2.10), the analytical solution for $v$ and $w$ seems to be beyond reach. The details of the finite difference method used here can be found in Muthu et al. (2008) for obtaining the solution for $v$ and $w$. We take 20 units of distance from origin which are very large represent and may infinity. Hence we discretise the interval $[1,20]$ into $n$ subintervals with $n+1$ nodes. Each node is represented by $r_{i}=1+i h$, with $h=19 / n$ the step length, starting from the first node $r_{0}=1$ to the last node $r_{n}=20$. The values of the functions $v, w$ at $r_{i}$ are given by $v_{i}$ and $w_{i}$. The symmetric derivative formulae at the i'th node are given as below

$$
\begin{aligned}
& v_{i}^{\prime}=\frac{v_{i+1}-v_{i-1}}{2 h}, \quad v_{i}^{\prime \prime}=\frac{v_{i+1}-2 v_{i}+v_{i-1}}{h^{2}}, \\
& v_{i}^{\prime \prime \prime}=\frac{v_{i+2}-2 v_{i+1}+2 v_{i-1}-v_{i-2}}{2 h^{3}}, \\
& v_{i}^{i v}=\frac{v_{i+2}-4 v_{i+1}+6 v_{i}-4 v_{i-1}+v_{i-2}}{h^{4}} .
\end{aligned}
$$

Substituting these derivatives given in Eqs (3.1) into Eq.(2.9), we get

$$
t_{1, i} v_{i-2}+t_{2, i} v_{i-1}+t_{3, i} v_{i}+t_{4, i} v_{i+1}+t_{5, i} v_{i+2}=0
$$

where

$$
\begin{aligned}
& t_{1, i}=r_{i}^{4}-h r_{i}^{3}, \\
& t_{2, i}=-4 r_{i}^{4}+2 h r_{i}^{3}+h^{2}\left(a_{1} r_{i}^{4}-3 r_{i}^{2}\right)-\left(a_{2} r_{i}^{3}+3 r_{i}\right) \frac{h^{3}}{2}, \\
& t_{3, i}=6 r_{i}^{4}-2 h^{2}\left(a_{1} r_{i}^{4}-3 r_{i}^{2}\right)+\left(a_{3} r_{i}^{4}+a_{4} r_{i}^{2}-3\right) h^{4}, \\
& t_{4, i}=-4 r_{i}^{4}-2 r_{i}^{3} h+h^{2}\left(a_{1} r_{i}^{4}-3 r_{i}^{2}\right)+\left(a_{2} r_{i}^{3}+3 r_{i}\right) \frac{h^{3}}{2}, \\
& t_{5, i}=r_{i}^{4}+h r_{i}^{3} .
\end{aligned}
$$


The finite difference form of Eq.(2.10) is as follows

$$
s_{1, i} w_{i-2}+s_{2, i} w_{i-1}+s_{3, i} w_{i}+s_{4, i} w_{i+1}+s_{5, i} w_{i+2}=0
$$

where

$$
\begin{aligned}
& s_{1, i}=r_{i}^{4}-h r_{i}^{3}, \\
& s_{2, i}=-4 r_{i}^{4}+2 h r_{i}^{3}+\left(b_{1} r_{i}^{4}-r_{i}^{2}\right) h^{2}-\left(b_{2} r_{i}^{3}+r_{i}\right) \frac{h^{3}}{2}, \\
& s_{3, i}=6 r_{i}^{4}-2 h^{2}\left(b_{1} r_{i}^{4}-r_{i}^{2}\right)+h^{4}\left(b_{3} r_{i}^{4}+b_{4} r_{i}^{2}\right), \\
& s_{4, i}=-4 r_{i}^{4}-2 h r_{i}^{3}+\left(b_{1} r_{i}^{4}-r_{i}^{2}\right) h^{2}+\left(b_{2} r_{i}^{3}+r_{i}\right) \frac{h^{3}}{2}, \\
& s_{5, i}=r_{i}^{4}+h r_{i}^{3} .
\end{aligned}
$$

\subsection{Method of solution}

We take the following boundary conditions

$$
v_{0}=v(1)=\cos \beta_{0}=n_{2}, \quad v_{n}=0,\left.\quad D^{2} v\right|_{r=1}=0 \quad \text { and }\left.\quad D^{2} v\right|_{r=\infty}=0 .
$$

Evaluating (3.2) for different values of $i$ we obtain

$$
\begin{aligned}
& i=0: \quad t_{1,0} v_{-2}+t_{2,0} v_{-1}+t_{4,0} v_{1}+t_{5,0} v_{2}=-t_{3,0} v_{0}, \\
& i=1: \quad t_{1,1} v_{-1}+t_{3,1} v_{1}+t_{4,1} v_{2}+t_{5,1} v_{3}=-t_{2,1} v_{0}, \\
& i=2: \quad t_{2,2} v_{1}+t_{3,2} v_{2}+t_{4,2} v_{3}+t_{5,2} v_{4}=-t_{1,2} v_{0}, \\
& i=3: \quad t_{1,3} v_{1}+t_{2,3} v_{2}+t_{3,3} v_{3}+t_{4,3} v_{4}+t_{5,3} v_{5}=0, \\
& i=n-1: t_{1, n-1} v_{n-3}+t_{2, n-1} v_{n-2}+t_{3, n-1} v_{n-1}+t_{5, n-1} v_{n+1}=-t_{4, n-1} v_{n}, \\
& i=n: \quad t_{1, n} v_{n-2}+t_{2, n} v_{n-1}+t_{4, n} v_{n+1}+t_{5, n-1} v_{n+2}=-t_{3, n} v_{n} .
\end{aligned}
$$

Thus the system of Eq.(3.7) represents $n+1$ equations in $n+3$ unknowns. Hence we require two more equations. These can be obtained from

$$
\left.D^{2} v\right|_{r=1}=0 \quad \text { and }\left.\quad D^{2} v\right|_{r=\infty}=0 .
$$


Using the boundary conditions (3.9), then we have

$$
t_{1} v_{-1}+t_{2} v_{1}=t_{3} v_{0} \quad \text { and } \quad t_{4} v_{n-1}+t_{5} v_{n+1}=t_{6} v_{n}
$$

where

$$
\begin{array}{lcc}
t_{1}=r_{0}^{2}-\frac{h r_{0}}{2}, & t_{2}=r_{0}^{2}+\frac{h r_{0}}{2}, & t_{3}=2 r_{0}^{2}+h^{2}, \quad t_{4}=r_{n}^{2}-\frac{h r_{n}}{2}, \\
t_{5}=r_{n}^{2}+\frac{h r_{n}}{2} & \text { and } & t_{6}=2 r_{n}^{2}+h^{2} .
\end{array}
$$

Expressing Eqs (3.7) and (3.8) in a matrix form as

$$
A_{1} X_{1}=B_{1}
$$

where

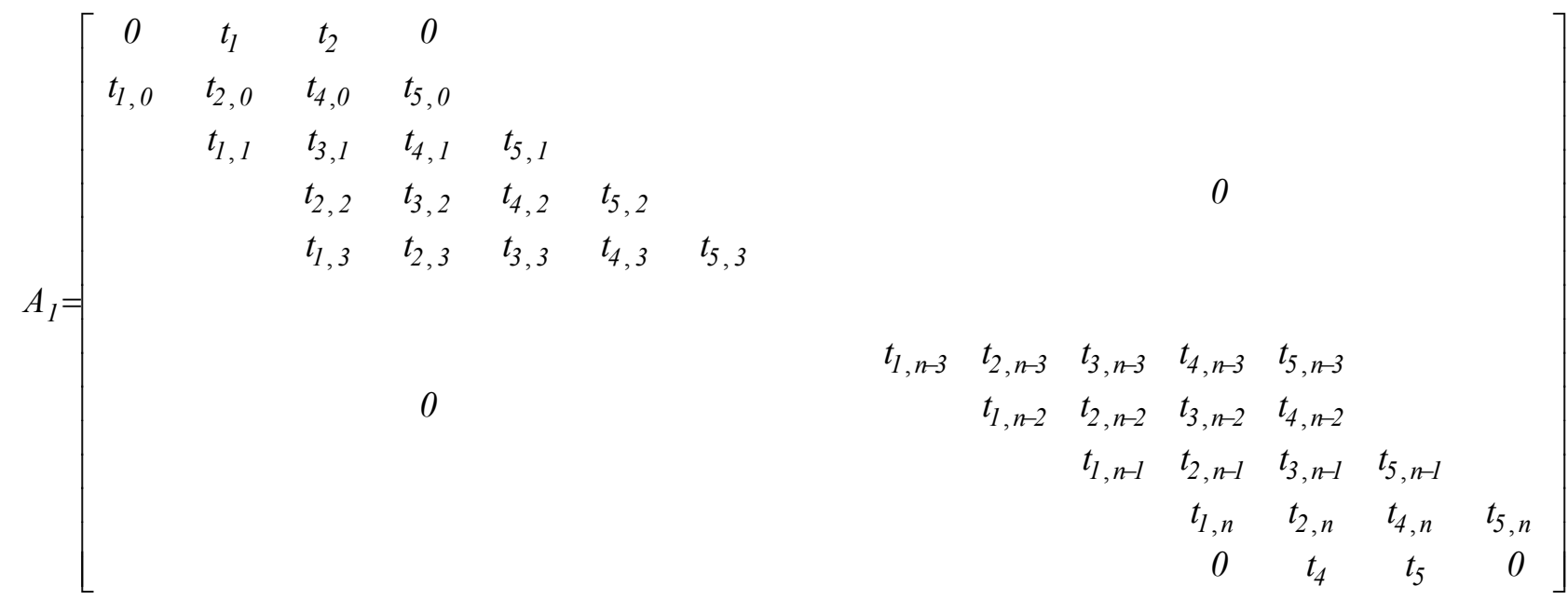

$$
\begin{aligned}
& X_{1}=\left[v_{-2}, v_{-1}, v_{1}, v_{2}, v_{3}, v_{4} \ldots \ldots \ldots v_{n-5}, v_{n-4}, v_{n-3}, v_{n-2}, v_{n-1}, v_{n}, v_{n+1}, v_{n+2}\right]^{T} \text { and } \\
& B_{1}=\left[t_{3} v_{0,}-t_{3,0} v_{0,}-t_{2,1} v_{0},-t_{1,2} v_{0}, 0 \ldots \ldots \ldots \ldots .,-t_{5, n-2} v_{n},-t_{4, n-1} v_{n},-t_{3, n} v_{n}, t_{6} v_{n}\right]^{T}
\end{aligned}
$$

where $v_{0}$ and $v_{n}$ are the values of $v$ on the boundary and are known and solving the system (3.10) we get the solution for $v$.

Now we find a solution for $w$ by applying the boundary conditions

$$
w_{0}=w(l)=\sin \beta_{0}=\sqrt{1-n_{2}^{2}}=n_{3}, \quad w_{n}=0, \quad w^{\prime \prime}-\left.e \frac{1}{r} w^{\prime}\right|_{r=1}=0 \quad \text { and } \quad w^{\prime \prime}-\left.e \frac{1}{r} w^{\prime}\right|_{r=\infty}=0 .
$$

Evaluating (3.4) for different values of $i$ we obtain 


$$
\begin{aligned}
& i=0: \quad s_{1,0} w_{-2}+s_{2,0} w_{-1}+s_{4,0} w_{1}+s_{5,0} w_{2}=-s_{3,0} w_{0}, \\
& i=1: \quad s_{1,1} w_{-1}+s_{3,1} w_{1}+s_{4,1} w_{2}+s_{5,1} w_{3}=-s_{2,1} w_{0}, \\
& i=2: \quad s_{2,2} w_{1}+s_{3,2} w_{2}+s_{4,2} w_{3}+s_{5,2} w_{4}=-s_{1,2} w_{0}, \\
& i=3: \quad s_{1,3} w_{1}+s_{2,3} w_{2}+s_{3,3} w_{3}+s_{4,3} w_{4}+s_{5,3} w_{5}=0, \\
& i=n-1: \quad s_{1, n-1} w_{n-3}+s_{2, n-1} w_{n-2}+s_{3, n-1} w_{n-1}+s_{5, n-1} w_{n+1}=-s_{4, n-1} w_{n}, \\
& i=n: \quad s_{1, n} w_{n-2}+s_{2, n} w_{n-1}+s_{4, n} w_{n+1}+s_{5, n-1} w_{n+2}=-s_{3, n} w_{n} .
\end{aligned}
$$

Thus the system of Eqs.(3.13) represents $n+1$ equation in $n+3$ unknowns. Hence we require two more equations which are obtained from the boundary conditions $w^{\prime \prime}-\left.e \frac{1}{r} w^{\prime}\right|_{r=1}=0$ and $w^{\prime \prime}-\left.e \frac{1}{r} w^{\prime}\right|_{r=\infty}=0$ , then we have

$$
s_{1} w_{-1}+s_{2} w_{1}=2 r_{0} w_{0} \quad \text { and } \quad s_{3} w_{n-1}+s_{4} w_{n+1}=2 r_{n} w_{n}
$$

where

$$
s_{1}=r_{0}+\frac{e h}{2}, \quad s_{2}=r_{0}-\frac{e h}{2}, \quad s_{3}=r_{n}+\frac{e h}{2} \quad \text { and } \quad s_{4}=r_{n}-\frac{e h}{2} .
$$

Expressing Eqs (3.13)-(3.14) in a matrix form as

where

$$
A_{2} X_{2}=B_{2}
$$

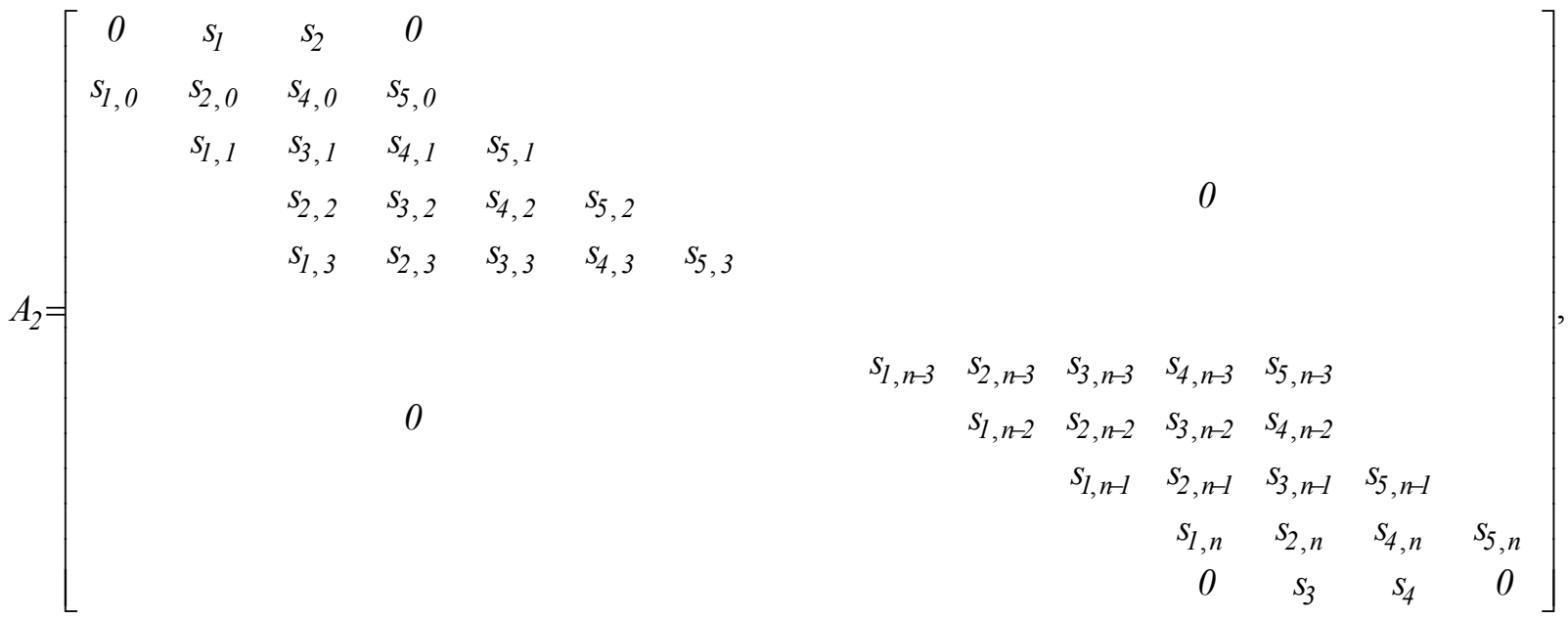

$$
\begin{aligned}
& X_{2}=\left[w_{-2}, w_{-1}, w_{1}, w_{2}, w_{3}, w_{4}, w_{5 . .} \ldots \ldots \ldots \ldots w_{n-5}, w_{n-4}, w_{n-3}, w_{n-2}, w_{n-1}, w_{n}, w_{n+1}, w_{n+2}\right]^{T} \text { and } \\
& B_{2}=\left[2 r_{0} w_{0},-s_{3,0} w_{0},-s_{2,1} w_{0},-s_{1,2} w_{0}, 0 \ldots \ldots \ldots 0,-s_{5, n-2} w_{n},-s_{4, n-1} w_{n},-s_{3, n} w_{n}, 2 r_{n} w_{n}\right]^{T}
\end{aligned}
$$

where $w_{0}$ and $w_{n}$ are the values of $w$ on the boundary and are known and solving the system (3.14) we get the solution for $w$. 


\section{Drag on the cylinder}

The drag $D$ acting on a cylinder of length $L$ is given by

$$
D=a L \int_{0}^{2 \pi}\left(T_{21} \cos \beta_{0}+T_{31} \sin \beta_{0}\right) d \theta .
$$

The stress component in Eq.(4.1) and couple stress tensor $M$ are defined by the following constitutive equations for couple stress fluids

$$
\begin{aligned}
& T_{i j}=-P I+\lambda\left(\nabla_{1} \cdot Q\right) I+\mu\left(\nabla_{1} Q+\left(\nabla_{l} Q\right)^{T}\right)+\frac{1}{2} I \times\left(\nabla_{l} \cdot M\right), \\
& M=m I+2 \eta \nabla_{l}\left(\nabla_{l} \times Q\right)+2 \eta^{\prime}\left[\nabla_{l}\left(\nabla_{l} \times Q\right)\right]^{T} .
\end{aligned}
$$

The stress components $T_{3 l}$ and $T_{2 l}$ on the cylinder can be calculated as

$$
\begin{aligned}
& T_{31}=\frac{\mu q_{0}}{a}\left[w^{\prime}+S\left(w^{\prime \prime \prime}+\frac{1}{r} w^{\prime}-\frac{1}{r^{2}} w\right)\right] e^{i \sigma_{2} t}, \\
& T_{21}=\frac{\mu q_{0}}{a}\left[v^{\prime}-\frac{v}{r}+S\left(v^{\prime \prime \prime}+\frac{2}{r} v^{\prime \prime}-\frac{1}{r^{2}} v^{\prime}+\frac{1}{r^{3}} v\right)\right] e^{i \sigma_{1} t} .
\end{aligned}
$$

Applying the finite difference scheme for Eqs (4.4) and (4.5), the non-dimensional form of stress components is calculated as

$$
\begin{aligned}
& T_{31}=\frac{\mu q_{0}}{a h^{3}}\left[-\frac{S}{2} w_{-2}+\left(\frac{-h^{2}}{2}+S+\frac{S h}{r_{0}}+\frac{S h^{2}}{2 r_{0}^{2}}\right) w_{-1}+\right. \\
& \left.-\frac{2 S h}{r_{0}} w_{0}+\left(\frac{h^{2}}{2}-S+\frac{S h}{r_{0}}-\frac{S h^{2}}{2 r_{0}^{2}}\right) w_{1}+\frac{S}{2} w_{2}\right] e^{i \sigma_{2} t}, \\
& T_{21}=\frac{\mu q_{0}}{a h^{3}}\left[-\frac{S}{2} v_{-2}+\left(\frac{-h^{2}}{2}+S+\frac{2 S h}{r_{0}}+\frac{S h^{2}}{2 r_{0}^{2}}\right) v_{-1}+\right. \\
& \left.+\left(\frac{S h^{3}}{r_{0}^{3}}-\frac{h^{3}}{r_{0}}-\frac{4 S h}{r_{0}}\right) v_{0}+\left(\frac{h^{2}}{2}-S+\frac{2 S h}{r_{0}}-\frac{S h^{2}}{2 r_{0}^{2}}\right) v_{1}+\frac{S}{2} v_{2}\right] e^{i \sigma_{1} t} .
\end{aligned}
$$

The non-dimensional drag can be calculated from Eq.(4.1) as

$$
D^{\prime}=T_{31} \cos \beta_{0}+T_{21} \sin \beta_{0}
$$

where

$$
D^{\prime}=\frac{D h^{3}}{2 L \pi \mu q_{0}}
$$




\section{Results and discussions}

The analytical expressions for the non-dimensional velocity components $v, w$ and drag are given by Eqs (2.9), (2.10) and (4.6) respectively. These values depend on the values of $\beta_{0}$, if $\beta_{0}=0$, we get only torsional oscillations and if $\beta_{0}=\pi / 2$, we get only axial (longitudinal) oscillations.

The numerical results are presented in the form of graphs for $S=10, \operatorname{Re}=0.1, \sigma_{1}=0.25, \sigma_{2}=0.5$, $\beta_{0}=0.7, n_{1}=0.6, t=\pi, e=0.5, \mathrm{Ha}=1$. The velocities $v$ and $w$ at different Reynolds number are shown in Figs 23 . We notice that as the Reynolds number Re increases both the velocities $v$ and $w$ decreases near the cylinder up to double the distance of the radius of the cylinder then are increasing as the distance increase. From Figs 4-5, we see that as the couple stress parameter $S$ increases, the transverse velocity $v$ and $w$ increases. Figures 6 and 7 show the effect of $e$, which is the ratio of couple stress viscosity coefficients $\eta$ and $\eta '$. It can be observed from these figures that the axial velocity increases near the cylinders and transverse velocity is insignificant with $e$. Figures 8 to 9 illustrate the effect of the magnetic parameter Ha on $v$ and $w$. It can be observed from these figures that the velocities $v$ and $w$ decrease with an increase in the parameter Ha. This happens because the imposed a magnetic field is perpendicular to the flow direction. This magnetic field gives rise to a resistive force and slows down the movement of the fluid. The velocities $v$ and $w$ at different non-dimensional times are shown in Figs 10-11. We observe that the transverse and axial velocity components near the cylinder are developing and fluctuating around zero with the same frequency as the cylinder. At the start of a cycle, the flow has its maximum velocities located at the surface of the cylinder, with a gradual decrease toward zero in the region away from the cylinder. As the cycle continues, the velocities decrease with the maximum values no longer at the cylinder surface but inside the flow field.

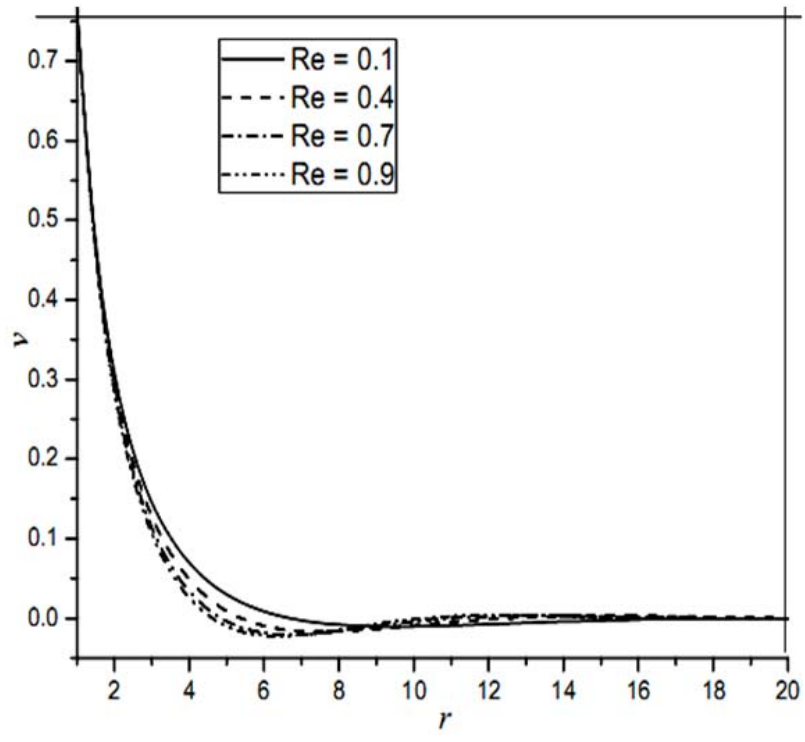

Fig.2. The effect of Re on velocity $v$.

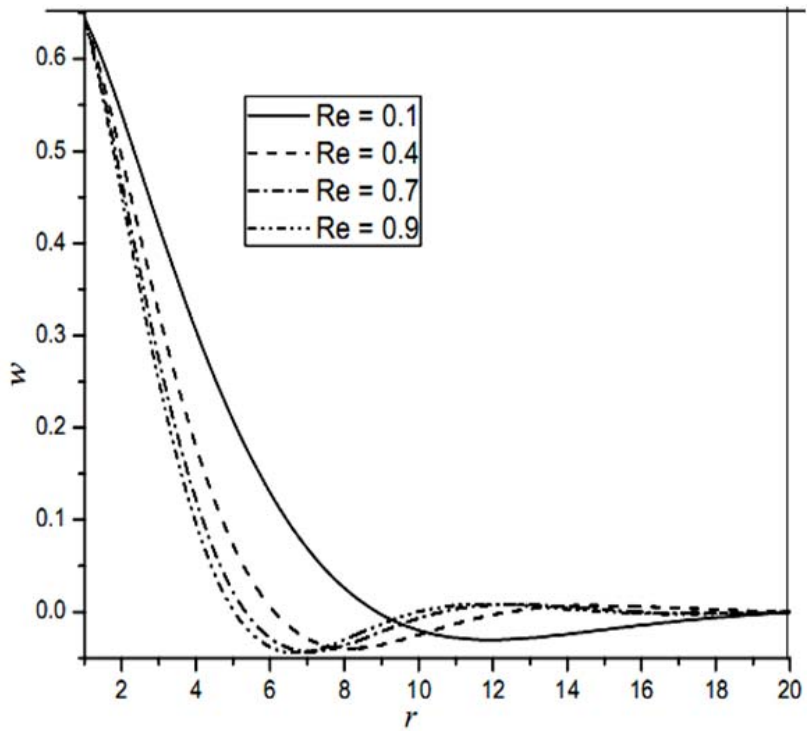

Fig.3. The effect of Re on velocity $w$. 


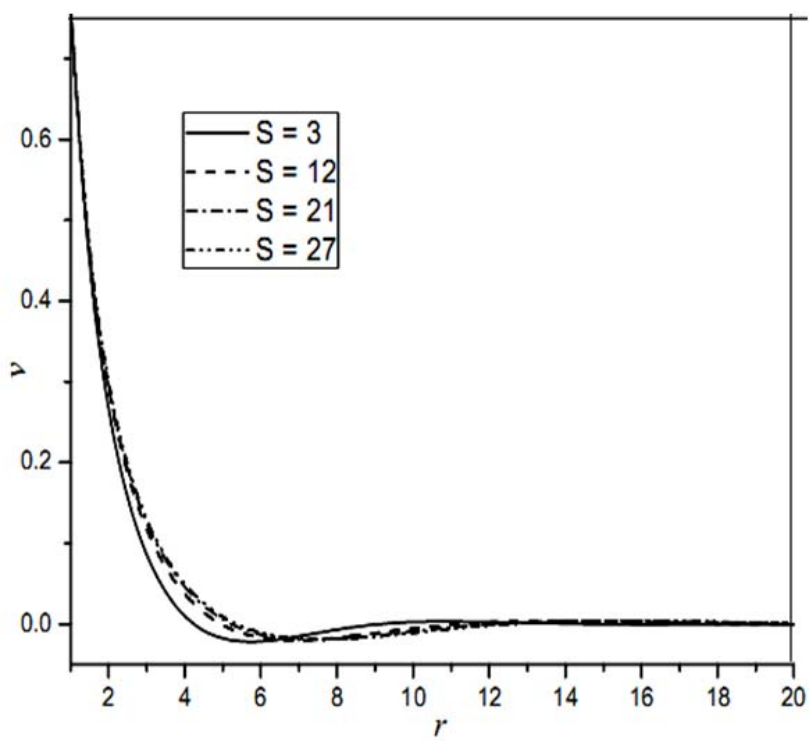

Fig.4. The effect of $S$ on velocity $v$.

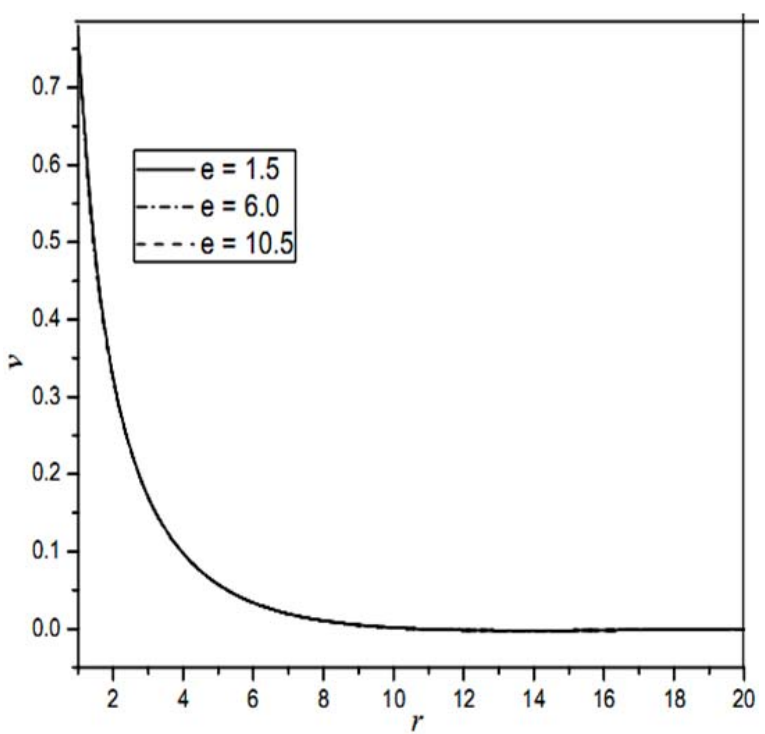

Fig.6. The effect of $e$ on velocity $v$.

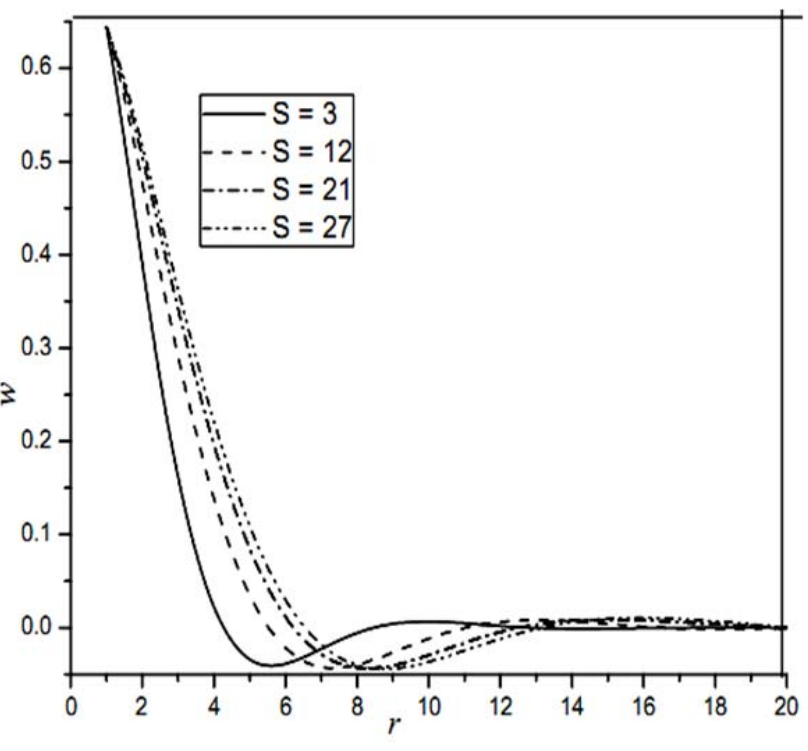

Fig.5. The effect of $S$ on velocity $w$.

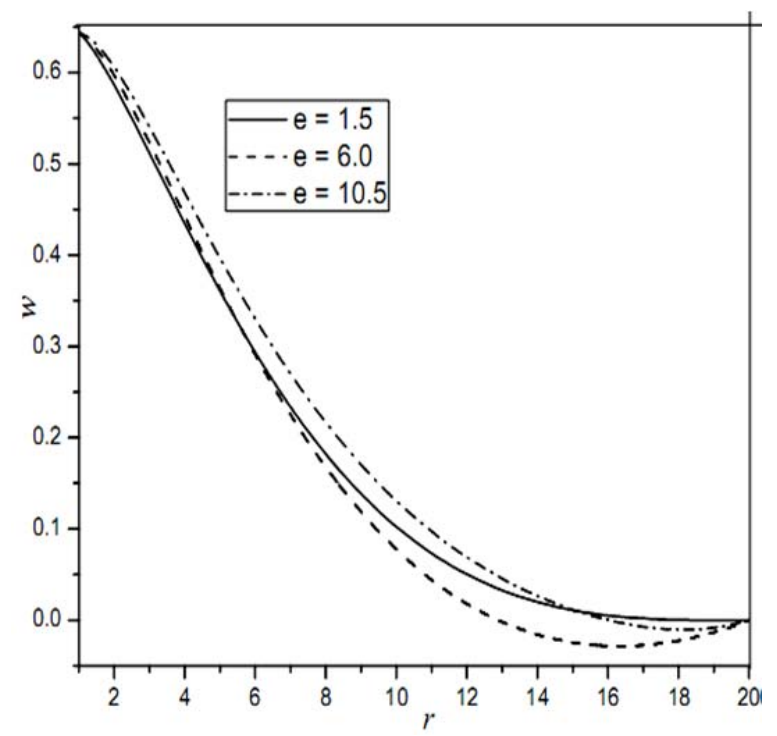

Fig.7. The effect of $e$ on velocity $w$. 


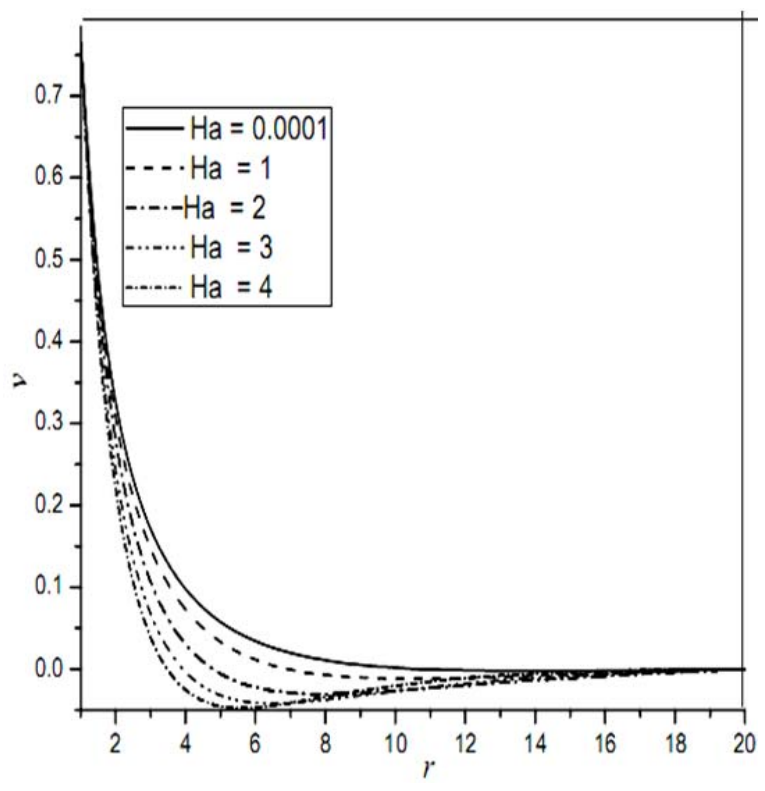

Fig.8. The effect of Ha on velocity $v$.

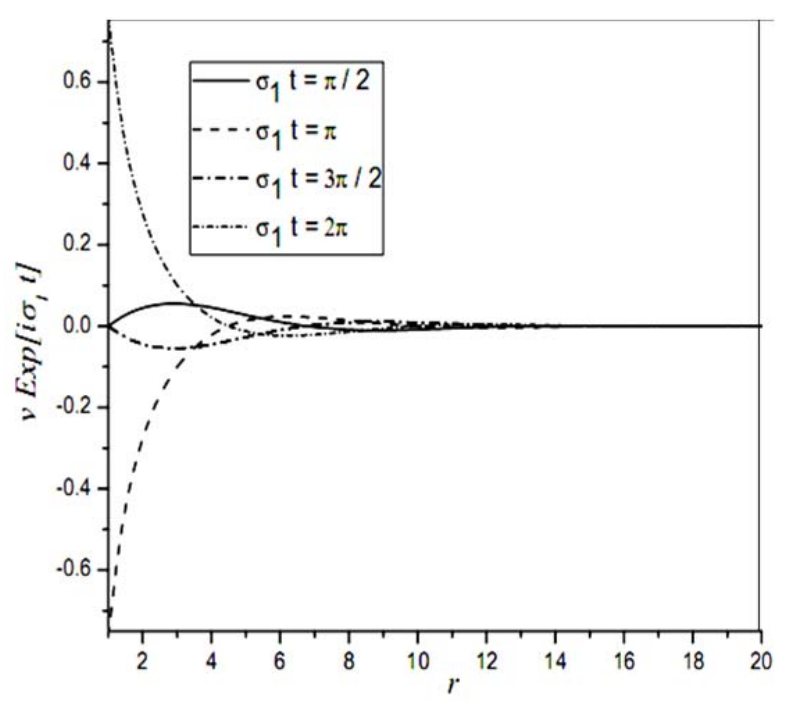

Fig.10. The effect of $\sigma_{l} t$ on velocity $v \operatorname{Exp}\left[i \sigma_{l} t\right]$.

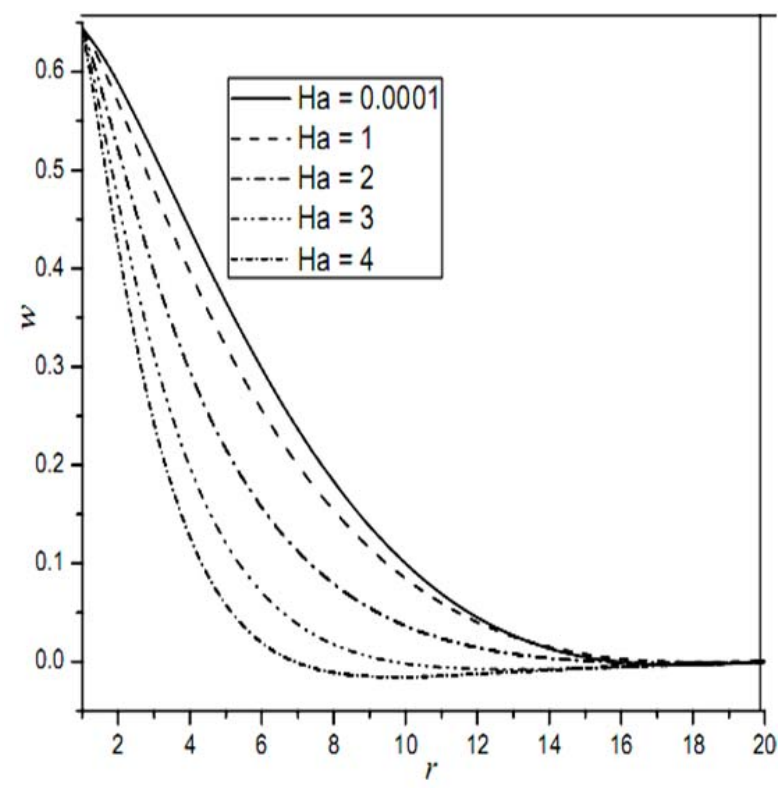

Fig.9. The effect of Ha on velocity $w$.

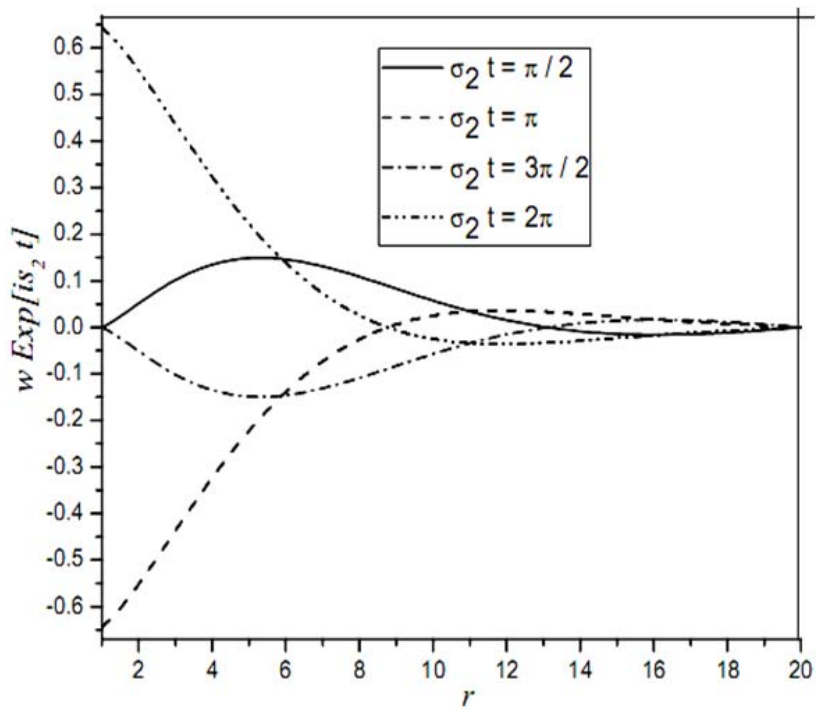

Fig.11. The effect of $\sigma_{2} t$ on velocity $v \operatorname{Exp}\left[i \sigma_{2} t\right]$.

The non-dimensional drag is calculated numerically for different values of non-dimensional time in multiples of $\pi / \sigma_{2}$ at fixed values of $\sigma_{1}, \sigma_{2}$ and the results are shown in Fig.12. In the equations of motion, the local acceleration term dominates if $\sigma_{1}, \sigma_{2}$ are large. To have all terms of LHS in the same order in Eqs (2.7) and (2.8), the frequency parameters $\sigma_{1}, \sigma_{2}$ are to be small. Hence we take both $\left|\sigma_{l}\right|,\left|\sigma_{2}\right|<1$. In calculation of drag also we observe that if $\left|\sigma_{1}\right|<1,\left|\sigma_{2}\right|<1$, the drag will be within reasonable values. From Fig.12, it can be seen that as the couple stress parameter $S$ increases, the amplitude of oscillations for drag increases. It can be seen from Fig.13, as $\sigma_{l}$ increases, the variations in drag at the cylinder wall are changing in amplitude and frequency. From Fig.14, it is observed that the drag oscillates irregularly as $\sigma_{2}$ increases. From Fig.15, we note that as the Reynolds number Re increases the magnitude of drag increases for small values of suction; but for higher values of suction the drag increases for small values of the Reynolds number and then 
decreases at higher values and is almost constant for very high values of the suction rate. From Fig.16, we note that drag decreases with the increasing values of $e$.

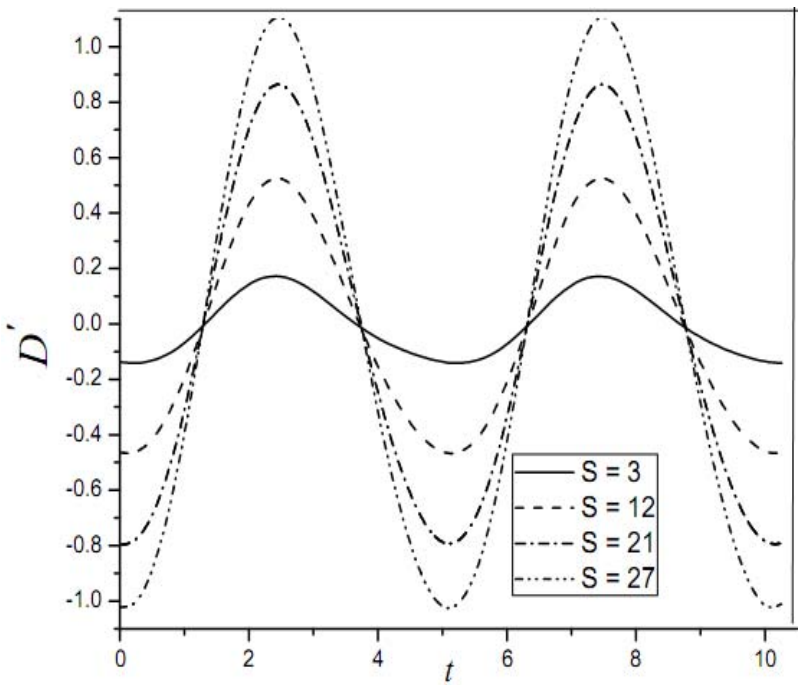

Fig.12. The effect of $S$ on drag.

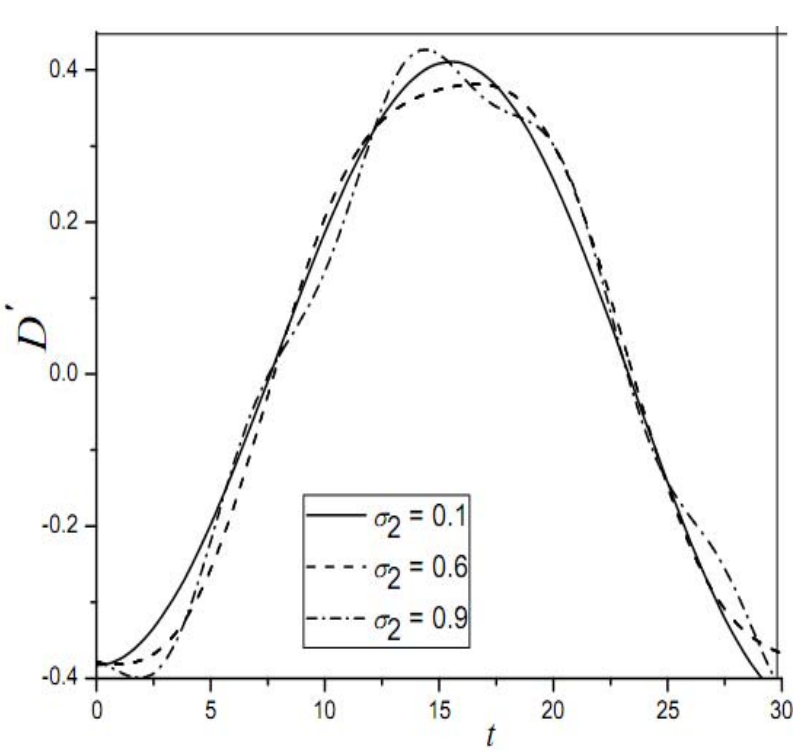

Fig.14. The effect of $\sigma_{2}$ on drag.

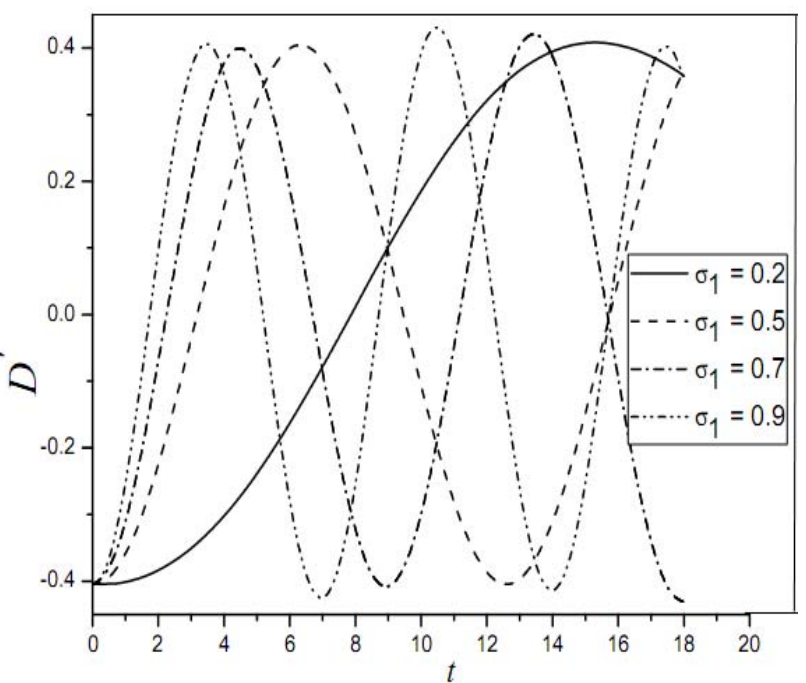

Fig.13. The effect of $\sigma_{l}$ on drag.

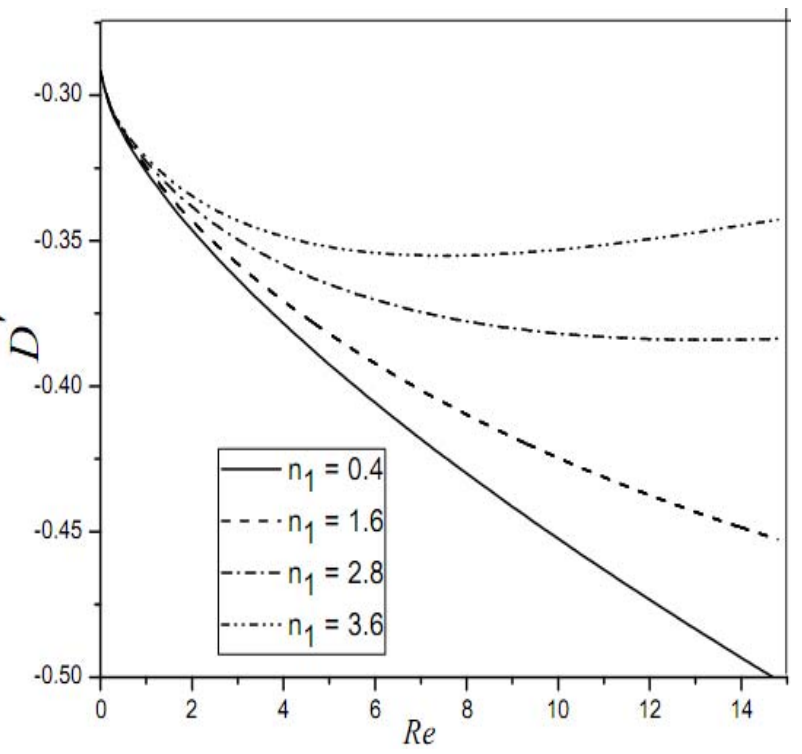

Fig.15. The effect of $n_{1}$ on drag. 


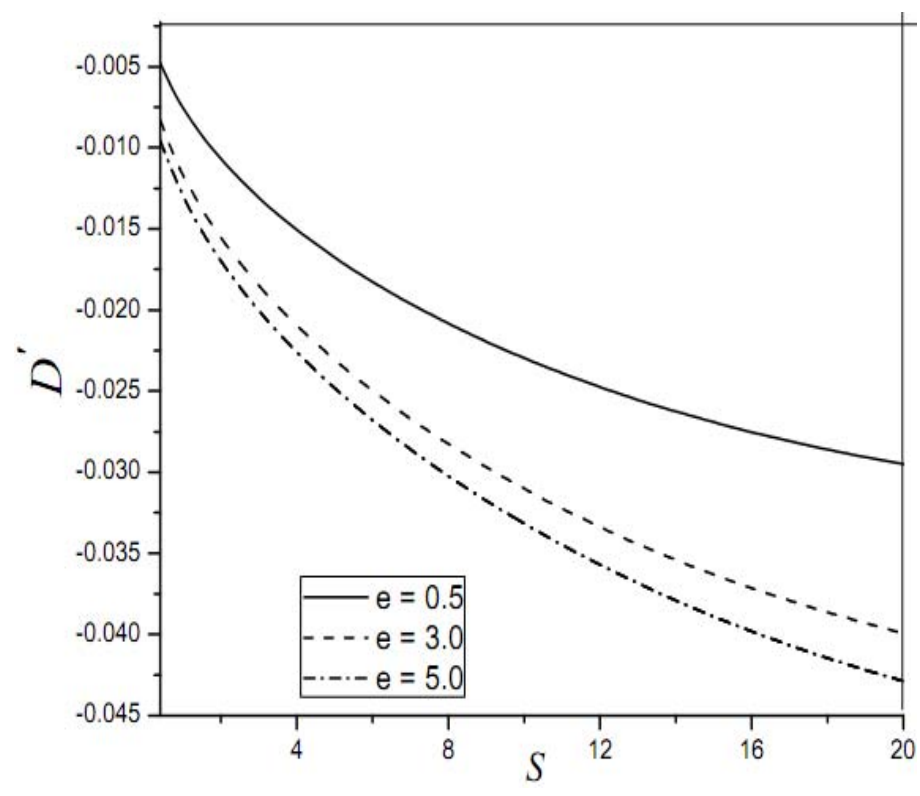

Fig.16. The effect of $e$ on drag.

\section{Conclusions}

In this paper, the radial magnetic field effect on the flow of the couple stress fluid generated by performing longitudinal and torsional oscillations and subjected to constant suction velocity at the surface of the cylinder is discussed. The velocity components are obtained numerically by applying the central finite difference scheme. From the present study we see that the presence of a magnetic field decreases both the axial and transverse velocities. The presence of couple stresses in the fluid increases both the velocities. It is also noted that the drag increases as $S$ increases i.e., the drag offered by viscous fluids is less than that of couple stress fluids and suction on the cylinder decreases the drag.

\section{Nomenclature}

$$
\begin{aligned}
a & - \text { radius of the cylinder } \\
B & - \text { magnetic field intensity } \\
B_{0} & - \text { magnitude of constant magnetic field } \\
C_{f} & - \text { coefficient of skin friction. } \\
D, D^{\prime} & - \text { dimensional and non-dimensional drag } \\
E & - \text { electric field strength } \\
e=\frac{\eta^{\prime}}{\eta} & - \text { ratio of couple stress viscosity coefficients } \\
e_{i j} & - \text { strain rate tensor } \\
e_{r}, e_{\theta}, e_{z} & - \text { unit base vectors } \\
\text { Ha } \sqrt{\frac{\sigma_{e} B_{0}^{2}}{\mu}} & - \text { Hartman number or magnetic parameter } \\
I & - \text { identity matrix } \\
J & - \text { current density } \\
L & - \text { length of the cylinder } \\
M & - \text { couple stress tensor } \\
m-1 / 3 & - \text { trace of } M \\
N_{2} & - \text { cos } \beta_{0}
\end{aligned}
$$




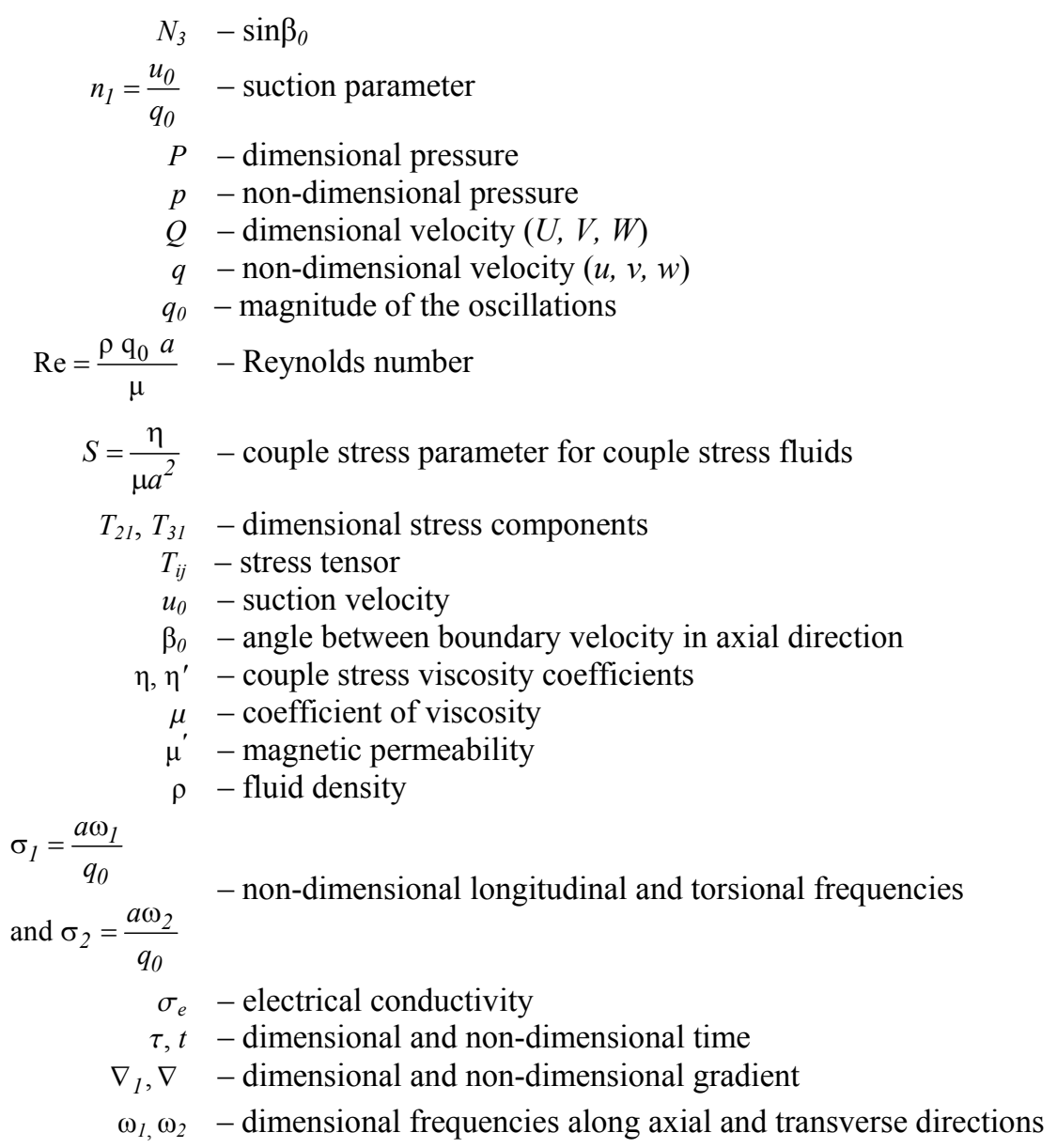

\section{References}

Agarwal H.L. (1969): Laminar flow through a uniform circular pipe with small outward normal suction. - Proc. Natn. Acad. Sci., vol.35, A, No.4, pp.518-527.

Bansal J.L. (1967): Laminar flow through a uniform circular pipe with small suction. - Proc. Natn. Acad. Sci., vol.32, A, No.4, pp.368-378.

Calmelet-Eluhu C. and Mazumdar D.R. (1998): Flow of micropolar fluid through a circular cylinder subject to longitudinal and torsinal oscillations. - Mathl. Comput. Modelling, vol.27, pp.69-78.

Hughes W.F. and Young F.J. (1966): Electro-Magnetodynamics of fluids.-John Villey: pp.254-256.

Muthu P., Rathish Kumar B.V. and Peeyush C. (2008): Peristaltic motion of micropolar fluid in cylindrical tubes: effect of wall properties. - Appl. Math. Modelling, vol.32, pp.2019-2033.

Owen D. and Rahaman K. (2006): On the flow of an Oldroyd-B liquid through a straight circular tube performing longitudinal and torsional oscillations of different frequencies. - Mathematica, vol.14, pp.1-9.

Pontrelli G. (1997): Longitudinal and torsional oscillations of a rod in an Oldroyd-B fluid with suction or injection.Acta Mech., vol.123, pp.57-68.

Ramana Murthy J.V., Bahali N.K. and Srinivasacharya D. (2010): Unsteady flow of a micropolar fluid through a circular pipe under a transverse magnetic field with suction/injection-Selguk. - Journal of Applied Mathematics, vol.11, No.2, pp.13-25. 
Ramana Murthy J.V., Nagaraju G. and Muthu P. (2010): Numerical solution of longitudinal and torsional oscillations of a circular cylinder with suction in a couple stress fluid. - Journal of Engineering and Applied Sciences, vol.5 No.5, pp.51-63.

Ramkissoon H. and Majumdar S.R. (1990): Flow due to the longitudinal and torsinal oscillations of a cylinder. ZAMP, Vol 41, pp 598-603.

Ramkissoon H., Easwaran C.V. and Majumdar S.R. (1991): Longitudinal and torsional oscillation of a rod in a polar fluid. - Int. J. Engng. Sci, vol.29(2), pp.215-221.

Singh D. and Rizvi S.A.T. (1964): Unsteady motion of a conducting liquid between two infinite coaxial cylinders.Phys. Fluids, vol.7, pp.760-761.

Srinivasacharya D. and Kaladhar K. (2012): Analytical solution of mixed convection flow of couple stress fluid between two concentric cylinders with Hall and Ion effects.-Turkish Journal of Engineering and Environmental Studies, pp.1-10 (To Appear).

Stokes V.K. (1966): Couple stresses in fluids. - Phys. Fluids, vol.9, pp.1709-1715.

Terril R.M. and Shrestha G.M. (1965): Laminar flow through parallel and uniformly porous walls of different permeability. - ZAMP, vol.16, pp.470-482.

Tsangaris S., Kondaxakis D. and Vlachakis N.W. (2007): Exact solution for flow in a porous pipe with unsteady wall suction and/or injection. - Communications in Nonlinear Science and Numerical Simulation, vol.12, pp.1181-1189.

Received: August 13, 2012

Revised: July 16, 2013 